EESTI NSV TEADUSTE AKADEEMIA TOIMETISED. XV KÖIDE FUOSIKA-MATEMAATIKA- JA TEHNIKATEADUSTE SEERIA. 1966, NR. 1

ИЗВЕСТИЯ АКАДЕМИИ НАУК ЭСТОНСКОН ССР. ТОМ ХV СЕРИЯ ФИЗИКО-МАТЕМАТИЧЕСКИХ И ТЕХНИЧЕСКИХ НАУК. 1966, № 1

Х. СУЙ, Ю. ИВАНОВ

\title{
ВЗАИМОДЕЙСТВИЕ СОИЗМЕРИМЫХ СТРУЙ В ОГРАНИЧЕННОМ ПРОСТРАНСТВЕ
}

Аэродинамические процессы, происходящие в топках паровых котлов, печах и различных камерах сгорания, определяют ход процесса горения топлива. Поэтому изучение движения и перемешивания газовых потоков в камерах является одним из существеннейших вопросов, интересующих многих исследователей.

В камерах сгорания одновременно протекает ряд сложных физико-химических процессов, каждый из которых сам по себе также достаточно сложен. Несмотря на исследования, проводимые в различных лабораториях многих стран, до сих пор еще не разработаны научные основы расчета этих процессов и в первую очередь аэродинажики камер.

В настоящей работе рассматриваются некоторые варианты экспериментального исследования на модели процессов перемешивания в камере прямоугольной формы при подаче в нее четырех воздушных изотермических струй.

Камера экспериментальной установки, в которой проводилось исследование перемешивания струй, оформлена в виде модели топки сланщевого котла ТП-17 (масштаб 1:20). Топочная камера этого котла с соотношением сторон около $1: 1,4$ оборудована четырьмя пылевыми горелками углового расположения, помещенными попарно на ее боковых (меныших) стенах.

Задача исследования состояла в выяснении характера движения потоков в камере при различных схемах подачи струй, приведенных схематически на рис. 1. Исследовались варианты подачи струй из сопел,

\section{1. Тангениалоная схема}
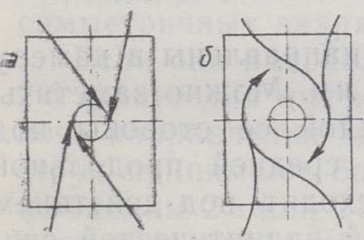

3 Блочная схема
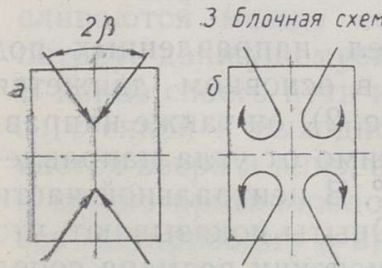
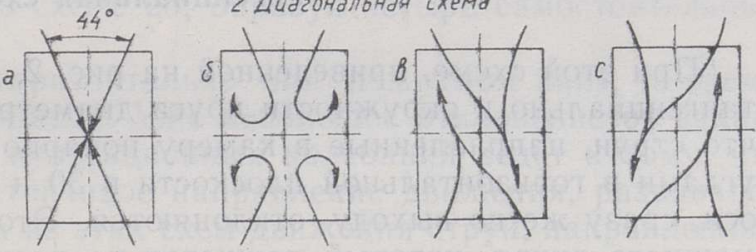
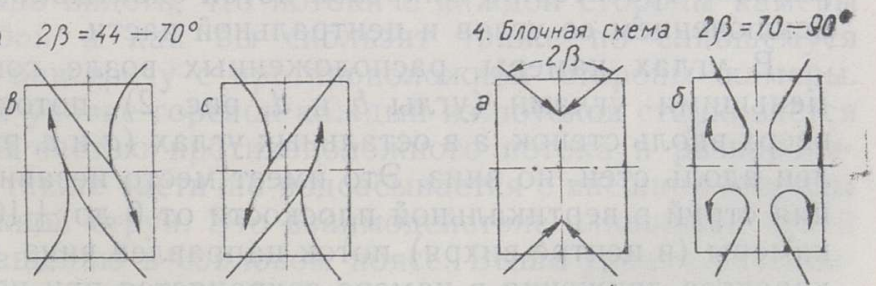

Рис. 1. Схемы различных вариантов направления и движения струй в камере.

6 ENSV TA Toimetised F-1 66 
расположенных по трем схемам: тангенциалыной, диагональной и блочной. Для осушествления этих вариантов, сопла имели возможность поворачиваться в горизонтальной и вертикальной плоскостях на некоторый угол.

Характер движения потоков и образование вихрей в камере определялись по зарисовкам, фотоснимкам, киносъемкой визуализированных потоков, а также по температурным измерениям в камере. По полям температур, замеренным при помощи электротермометра в четырех различных горизонтальных сечениях по высоте модели, можно было судить и о процессах перемешивания в топочной камере котла при различных вариантах подачи струй.

В камере на уровне сопел искусственно создавалось перавномерное температурное поле. Для этой цели в каждом опыте две соседние струи слегка подогревались. Разница температур между холодными и нагретыми струями составляла $17^{\circ}$.

Предварительными опытами было установлено, что на окончательный результат процесса перемешивания не влияет, какие две струи из четырех нагреваются. Также было проверено, что на процесс перемешивания не влияет величина температуры подогрева.

Сопла были изготовлены аналогично одному из вариантов пылевых горелок, установленных на котлах ТП-17. Каждое сопло, имея внешние размеры $25 \times 123$ мм, составлялось из трех находящихся одно над другим самостоятельных сопел. Размеры свободного сечения каждого отдельного сопла составляли: в основном варианте $-22 \times 38$ мм (большие сопла) и в дополнительном варианте - $15,6 \times 31,7$ мм (малые сопла), образованные вставками в большие. Дополнительный вариант с малыми соплами был выполнен для проверки влияния размера сопел или величины выходной скорости на характер протекания процессов в камере.

Средняя выходная скорость больших струй была 21,6 и малых струй при том же расходе 36,6 м/сек. При сохранении размера сопел, изменение выходной скорости в пределах $17-25$ м/сек не изменяло характера движения потоков в камере.

Ниже приводятся результаты исследований.

\section{Тангенциальная схема}

При этой схеме, приведенной на рис. 2, сопла направлены в камеру тангенциально к окружности круга диаметром 80 мм. Можно заметить, что струи, направленные в камеру попарно с каждой ее стороны под углами в горизонтальной плоскости в 30 и $13^{\circ}$ от средней продольной оси, сразу же по выходу отклоняются. Это происходит под действием мощного вихря, который движется по восходящей эллиптической спиральной траектории. Вихревой поток охватывает все сечение камеры, за исключением ее углов и центральной части.

В углах камеры, расположенных возле сопел, направленных под меньшими углами (углы $b$ и $d$, рис. 2), поток в основном движется вверх вдоль стенок, а в остальных углах ( $a$ и $c$, рис. 2) он также направлен вдоль стен, но вниз. Это имеет место независимо от угла направления струй в вертикальной плоскости от 0 до $\pm 10^{\circ}$. В центральной части камеры (в центре вихря) поток направлен вниз. Опыты показывают, что характер движения в камере сохраняется при изменении размера сопел с большего на меньший. Установлено также, что при подаче того. 

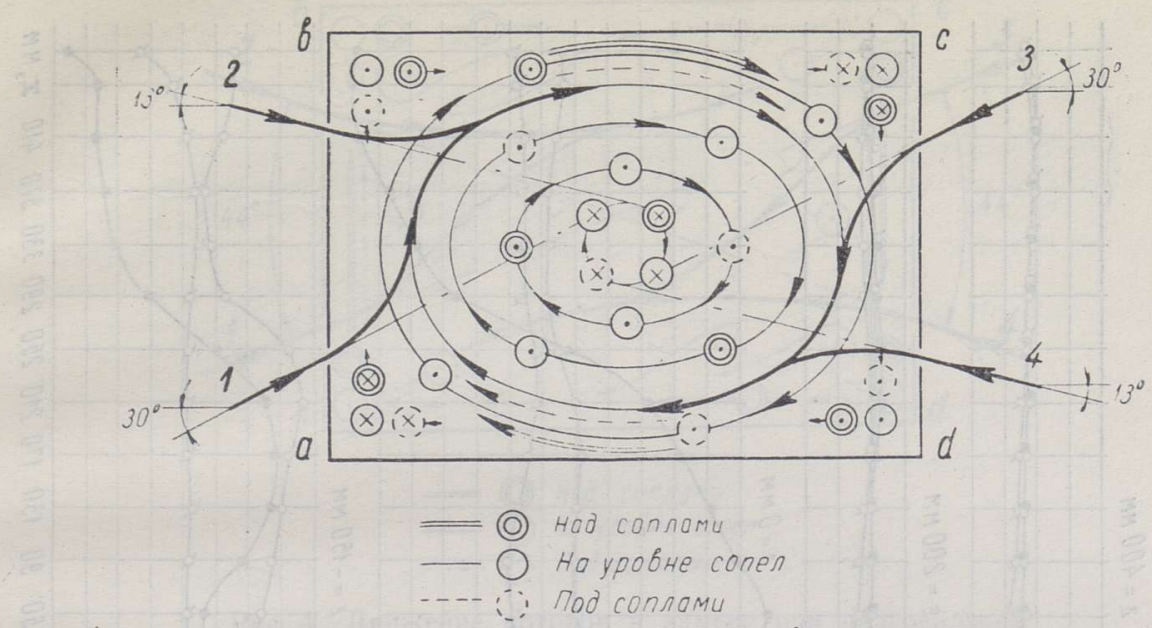

Рис. 2. Движение потоков в камере при тангенциальной схеме.

же расхода из сопел меньшего размера процесс перемешивания протекает несколько лучше.

Температурные поля в координатах $x ; t-t_{1}(t-$ температура в измеряемой точке, $t_{1}$ - температура холодного воздуха), построенные для различных сечений по высоте камеры, позволяют судить о процессе перемешивания в ней. На рис. 3 каждое сечение характеризуется тремя температурными линиями, параллельными фронтовой стенке камеры, на расстояниях $y=80,180$ и 280 мм от нее. Приведенные кривые свидетельствуют о том, что при тангенциальной схеме подачи струй. несмотря на интенсивное движение потоков в камере, процесс полного перемешивания до выхода из камеры все же не обеспечен.

\section{Диагональная схема}

При этой схеме струи, направленные в камеру по ее диагоналям, могут развиваться по трем различным схемам движения (см. рис. 1, схемы $2 a$, б и в). Струи, направленные в камеру под углом вверх, развиваются в дальнейшем по схеме 26 , образуя четыре самостоятелыных симметричных вихря.

Если они направлены горизонтально или под углом вниз, то схема 26 станет для них неустойчивой. Уже небольшое отклонение от заданного направления за счет пульсационных колебаний ведет к тому, что струи принимают более устойчивое направление движения, развиваясь по схемам 2 в или 2 г. Одна из этих схем движения струй, направленных горизонтально в камеру по диагональной схеме, показана на рис. 4. Из этого рисунка можно видеть, что потоки с каждой стороны камеры сливаются между собой и как бы скользят также по слившемуся потоку, движущемуся навстречу с противоположной стороны камеры. В конце своего пути на уровне горелок каждый из потоков сталкивается со стенкой и выходной частью противоположного потока и разворачивается вверх и вниз, а также частично подсасывается с внешней стороны к корню противоположной струи. Это взаимодействие слившихся струй все же приводит к вращению в сопловом поясе. Выше уровня установки соплового пояса поток приобретает более заметное общее вращение, но оно значительно слабее, чем при тангенциальной схеме. (Следует от- 


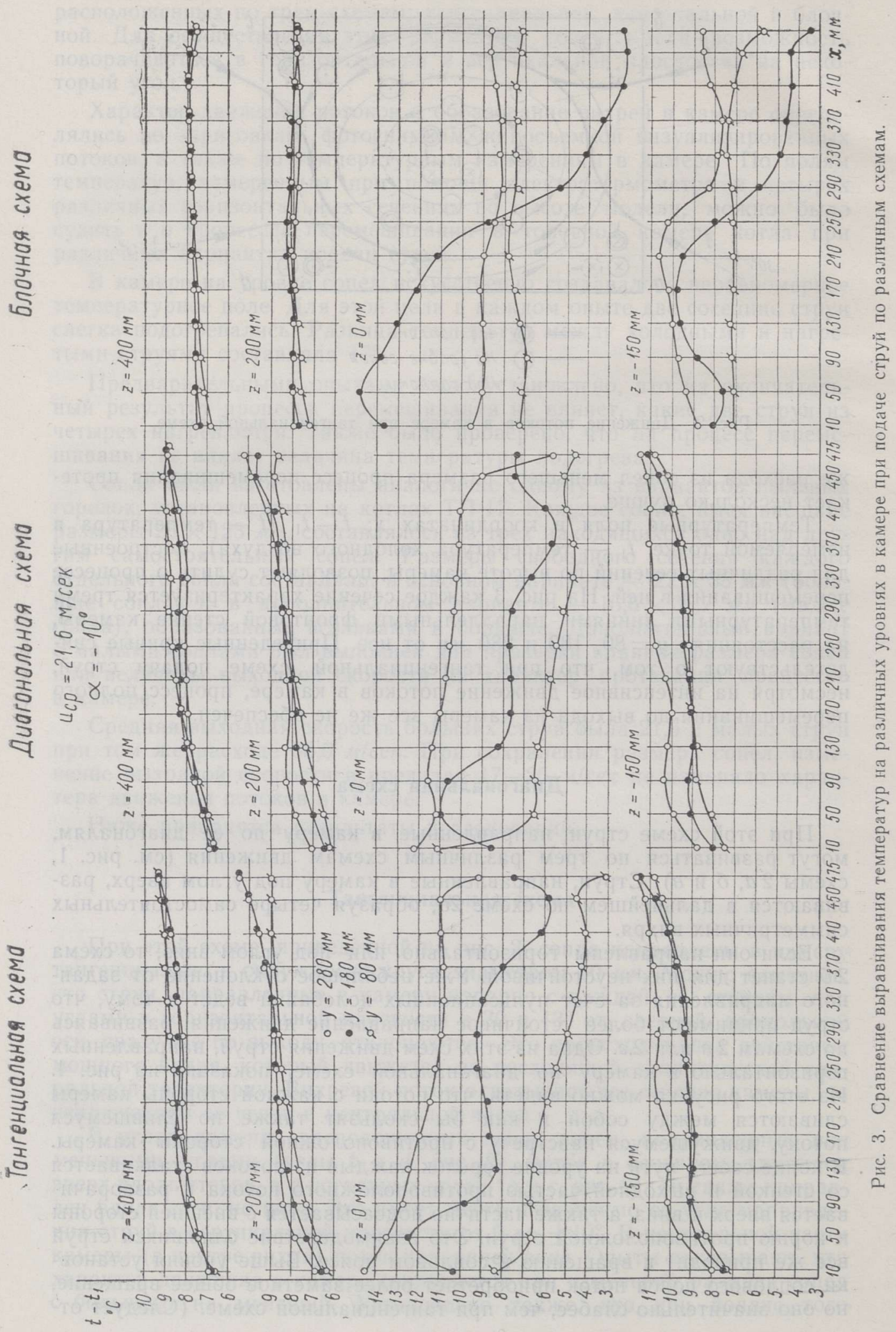




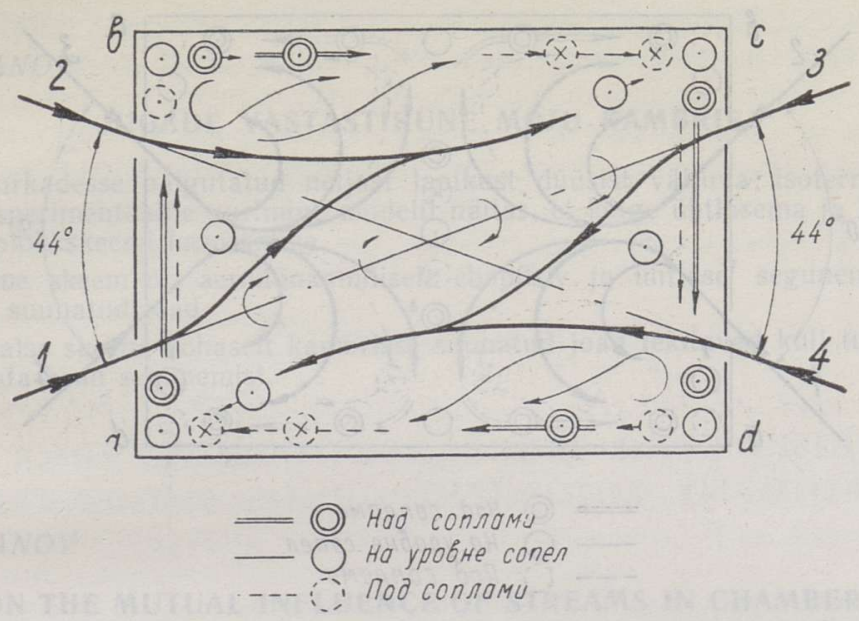

Рис. 4. Движение потоков в камере при диагональной схеме.

метить, что вращение может быть как правое, так и левое и может даже в течение процесса изменять свое направление.) В углах $b$ и $d$ потоки движутся вверх. Между соседними парными струями также образуются слабые восходящие потоки. Перемешивание потоков по высоте камеры неполное (см. рис. 3) и в некоторых случаях даже несколько хуже, чем при тангенциальной схеме.

При симметричной картине движения с образованием четырех вихрей (см. схему 2 б, рис. 1) процесс перемешивания существенно интенсифицируется, и температурные кривые сближаются и выравниваются. Движение струй в этом варианте аналогично симметричному варианту блочной схемы, более подробно описанному ниже.

Изменение размера сопел не влияет на характер движения потоков.

\section{Блочная схема}

При блочной схеме струи, выходящие из боковых стенок попарно, встречаются между собой под углами 60 и $90^{\circ}$ (см. схемы 3 и 4 , рис. 1 ). Фокусы (места встречи струй) на длинной оси камеры соответственно расположены на расстоянии в 0,68 и 0,36 полуширины модели (от места выхода струй).

При направлении струй блочной схемы вверх под углом $10^{\circ}$ в камере, несмотря на изменение места расположения фокусов на длинной оси, образуется симметричная картина движения потоков в виде четырех вихрей. Если струи направлены горизонтально или под углом $10^{\circ}$ вниз, то при изменении угла встречи от 90 до $70^{\circ}$ движение сохраняет симметричный характер по схеме 46 , изображенной на рис. 1. Опыты со струями под углом атаки $60^{\circ}$ и меньше, когда встреча струй происходит недалеко от центра, показали, что симметричная схема становится неустойчивой и течение становится в основном подобным движению при диагональной схеме (см. схемы 2 в, г или 3 в, г, рис. 1).

При блочной схеме симметричное движение четырех вихрей на уровне сопел изображено на рис. 5. Такая картина движения сохраняет устойчивость при всех режимах, независимо от значения угла поворота сопел в вертикальной плоскости, если угол встречи струи больше $70^{\circ}$ или расстояние от стенки до фокуса меньше $\sim 0,6$ полуширины камерьі. 


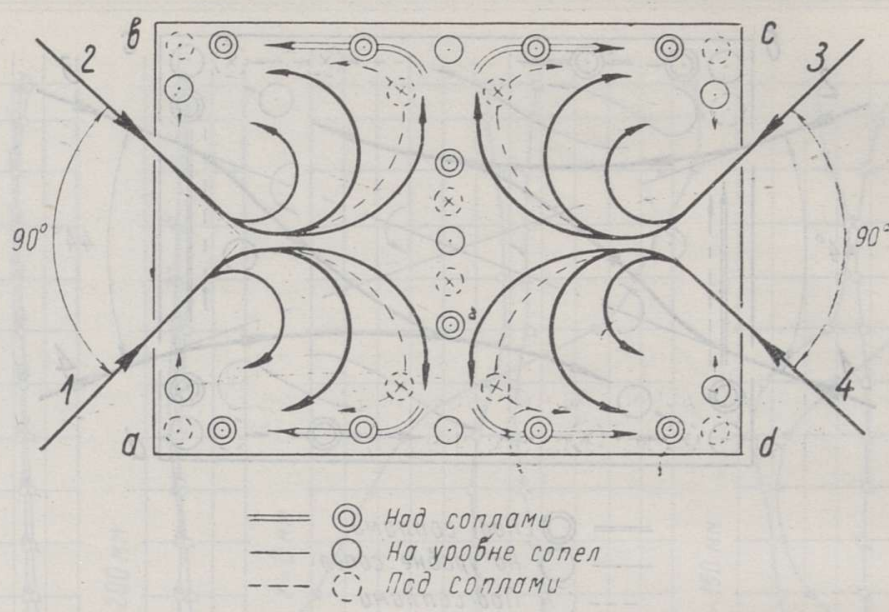

Рис. 5. Движение потоков в камере при блочной схеме.

Қаждый вихрь образован своей струей. На короткой (поперечной) осн камеры, т. е. в месте встречи противоположных потоков, над уровнем сопел движение происходит вверх, а ниже уровня сопел - вниз. Во всех углах камеры поток движется снизу вверх вдоль стен. Выше сопел влияние крупных вихрей уменьшается, и перемешанный поток движется вверх камеры, разбиваясь на вихри малых размеров. Перемешивание потоков при этой схеме подачи струй происходит лучше, и уже на половине пути до выходного сечения температурные поля выравниваются, как это видно на правых графиках рис. 3. При переходе на сопла меньшего размера общий характер движения потоков в камере сохраняется, но процесс перемешивания, как и следовало ожидать, несколько интенсифицируется.

Проведенные исследования позволяют сделать следующие основные выводы о течении потоков в камере при подаче трех вариантов соизмеримых струй:

1. Наиболее быстрое и равномерное перемешивание обеспечивается при подаче четырех струй по устойчивой блочной схеме.

2. Устойчивость блочной схемы обеспечена, если струи направленьі под углом $10^{\circ}$ вверх или расстояние фокусов меньше 0,6 полуширины камеры (угол встречи струй больше $70^{\circ}$ ).

3. Диагональная схема неустойчива. Ее можно стабилизировать направлением струй под углом $10^{\circ}$ вверх. В. этом случае обеспечивается равномерное перемешивание, примерно аналогичное блочной схеме.

4. Струи, направленные в камеру по тангенциальной схеме, образуют сильный вихрь во всем объеме камеры. Однако при этом не обеспечивается равномерное перемешивание потоков до выходного сечения камеры.

5. Исследования показали также, что при заданном угловом размещении горелочных устройств изменение размеров устья сопел и выходной скорости воздуха не влияют на аэродинамику камеры.

Институт термофизики и электрофизики Академии наук Эстонской ССР
Поступила в редакцию 2/III 1965 


\section{H. SUI, J. IVANOV}

\section{JUGADE VASTASTIKUNE MÕJU KAMBRIS}

Kambri nurkadesse paigutatud neljast lapikust düüsist väljuva isotermilise õhujoa segunemise eksperimentaalne uurimine mudelil näitas, et kõige ühtlasema ja kiirema segunemise tagab plokkskeemi kasutamine.

Diagonaalne skeem on aerodünaamiliselt ebapüsiv ja ühtlase segunemise tagavad vaid ülespoole suunatud joad.

Tar:gensiaalse skeemi kohaselt kambrisse suunatud joad tekitavad küll tugeva keerise, kuid ei kindlusta head segunemist.

\section{H. SLI, J. IVANOV}

\section{ON THE MUTUAL INFLUENCE OF STREAMS IN CHAMBER}

The experimental studies, on a model, of streams emerging from four flat jets placed into the corners of the chamber showed that the application of a block scheme affords the quickest and most uniform mixing.

A diagonal scheme is aerodynamically unstable, and a regular mixture is guaranteed only in case of upwards-directed streams.

The streams directed into the chamber according to a tangential scheme produce a strong whirl, but do not guarantee satisfactory mixing.- 